\title{
Authors' Response: the virtues of minimalism in ontology and epistemology
}

forthcoming in Metascience, Book symposium on Michael Esfeld and Dirk-André Deckert: A minimalist ontology of the natural world. New York: Routledge 2017. Pp. 182 US\$140.00HB, DOI: 10.1007/s110116-018-0322-2.

\section{Michael Esfeld}

University of Lausanne, Philosophy Department, Michael-Andreas.Esfeld@unil.ch, www.michaelesfeld.com

\section{Dirk-André Deckert}

Ludwig Maximilians University Munich, Department of Mathematics, deckert@math.lmu.de

\section{Ontology}

The book seeks to answer the following question: What is an ontology that is minimally sufficient to accommodate the known physics as well as our common sense knowledge about the natural world? The answer developed in the book is that the commitment to matter points individuated by distance relations and the change of these relations constitutes such a minimalist ontology. The issue is minimal sufficiency. There is no claim about necessity or $a$ priori. This ontology obviously is akin to Descartes' view of nature as res extensa, which can also be seen as a kind of minimal sufficiency claim, but we do not share Desscartes' concerns about necessity and a priori. Minimal sufficiency, not necessity means that there may be other proposals that are also minimally sufficient as an ontology of the natural world. We are open to this possibility (although, for our part, we are happy to have developed just one possibility). We agree with Alastair Wilson "that the correct ontology is the one which offers the best overall explanation of the facts - including the epistemological facts". That these 
facts boil down to evidence about relative positions of discrete objects and their change may be taken as motivation to try out the ontology proposed in the book; but it is not the argument for this ontology. The argument is that this ontology offers the overall best explanation: less commitments would deprive us of an explanation; more commitments would not lead to an explanatory gain, but only to trouble in the ontology.

Hence, the proposal set out in the book may be wrong-headed for three reasons:

a) The proposal is not minimally sufficient, that is, it contains redundant commitments: one can do with less ontological commitments.

b) The proposal is not sufficient, that is, more ontological commitments are required to accommodate the known physics as well as our common sense knowledge.

c) The proposal is overambitious, that is, physics (not to mention common sense) is too diverse so that no single ontology can accommodate the whole of physics.

The suspicion that the book is in fact engaged in a priori metaphysics and is after necessity derives from (c). Since most of today's philosophy of physics is concerned with the analysis of particular physical theories, the claim that there is a single ontology that covers the whole of physics may appear to be as old-fashioned as a priori and necessity claims are when it comes to scientific metaphysics. But this is just our claim: there is at least one fundamental ontology available that accommodates the whole of physics, and this claim is worked out in detail for classical, relativistic and quantum physics in the book. So the question is not whether this claim has a smell of a priori or necessity, but whether it achieves what it sets out to do, that is, whether it provides a coherent ontology for the existing physical theories. Of course, pursuing such a project requires taking a stance on controversial issues in the interpretation of physical theories, in particular when it comes to quantum physics. George Darby takes this to be a drawback of our project. But there is no ontology of the natural world based on science that remains neutral with respect to these controversies. In particular, any 
proposal for an ontology in the domain of quantum physics has to provide a solution to the measurement problem. The thesis argued for in the book is that the proposed minimalist ontology enables the formulation of a cogent solution of this problem.

In a certain sense, Anna Marmodoro takes issue with (a). Her concern is not that the ontology that we propose is too rich. She has reservations about us admitting three irreducible primitives: "matter points", "distances" and "change". Her comments can be taken to suggest that we could just do with distance relations as primitive and derive objects as well as change from these relations. However, as Marmodoro acknowledges, the ontic structural realism that we endorse is a moderate one. Its being moderate means that we accept both objects and relations as primitives. All there is to the objects is their standing in relations such that the relations individuate the objects. But letting relations individuate objects does not amount to deriving objects from relations, since relations still require relata as a primitive (see Esfeld and Lam 2011). We do not see how objects could be derived from relations (nor do we understand how, in a (neo-)Aristotelian metaphysics, relations could be derived from objects).

The issue then is whether, given objects individuated by relations, there is an answer to the following question: What is the origin of change? We postulate the change in the distance relations as a further primitive. Nonetheless, it may be possible to merge our two axioms in one by conceiving the distance relations in such a way that it is in their nature to change. We are open to this possibility, as we remain finally neutral on the metaphysics of modality (cf. p. 56 of the book). However, enriching the ontology by admitting primitive modal entities in the guise of powers would not answer the question why there is change at all. Powers are powers for a certain change to occur (their manifestation), that is, one particular change in contrast to other particular changes; but this does not answer the question why they manifest at all. Simply saying that is in the nature of powers to manifest would not be an illumniating answer 
to that question in the same way as it would not be an informative answer to the question why there is change to simply say that it is in the nature of the distance relations to change.

In any case, all that science gives us are distance relations connecting point particles and parameters that are introduced in terms of their functional role for the evolution of the distance relations. That is why the commitment to powers for particular changes does not lead to an explanatory gain. However, we have not always polished up the language that we use in line with the (Super-)Humeanism that is implicit in our axioms. We may occasionally speak of laws as governing, or of the wave function as acting on points in configuration space. But these are metaphorical ways of speaking. There is change, and there is our representation of change by means of laws, wave functions, etc. Our ontology does not have the burden to give an account of how laws or wave functions govern or "communicate" with physical systems.

We do not claim originality for our conception of materiality in terms of matter points individuated by distance relations and their change. We take it to be an elaboration on Desccartes' view of nature as characterized only by extension and motion, which laid the ground for modern science. Anna Marmodoro correctly points out that our conception of the distance relations goes far beyond the claim that everything in the natural world is spatially related to everything else. We subscribe to a substantial holism in taking these relations to provide for the identity of their relata. Nonetheless, we would not call this stance "geometrical holism", for two reasons: (a) the term "geometrical" may be misleading because the distance relations do not come with a particular geometry; (b) the issue are relations that individuate objects. But these do not have to be distances; as we mention in the book $(21,162)$, there may also be other relations, such as thinking relations individuating mind points. In brief, this holism just is (moderate) ontic structural realism, with the structures being the distance 
relations. There is nothing original in our conception of holism as a horizontal one, consisting in relations that individuate objects (see Pettit 1993, chs. 3-4, and Esfeld 1998).

This metaphysical holism is indeed distinct from the dynamical, or physical holism that is implemented in any fundamental physical theory due to the fact that its dynamical structure concerns the whole universe at once and subsystems of the universe only derivatively. This metaphysical holism paves the way for this dynamical holism in the sense that any change in the identity providing relations is a change that concerns all the elements in the system; as we point out in the book (40), this holds for instance also for semantic holism. However, what precise form this dynamical holism takes depends on the change that actually occurs. On Humeanism, the change comes first, and then comes the dynamical structure of a physical theory to represent that change in a manner that optizimes simplicity and informational content. Since we consider the change to be continuous, it provides for an identity of the matter points across all change, as there always is a non-vanishing distance between any two matter points. In a nutshell, we take permanent matter points with permanently changing distance relations to implement the best of both Parmenidian and Heraclitean metaphysics.

In making the commitment to a substantial, metaphysical holism, our position, dubbed SuperHumeanism, deviates from the standard, Lewisian Humeanism as well as from George Darby's Almost-Lewisian Humeanism. Indeed, it is more radical than these forms of Humeanism: the Lewis-style Humean is not only committed to point particles and distance relations, but also to intrinsic properties of the point particles (for which mass and charge may count as paradigm examples), and she may include fields in the mosaic if they can be conceived as intrinsic properties of points (but runs into notorious difficulties mentioned by Darby if she intends to include also the quantum state in the supervenience basis, since the quantum state cannot be construed as intrinsic properties of point objects, at least not in 
ordinary space). The argument for deleting the commitment to intrinsic properties consequent upon taking the matter points to be individuated by the distance relations is not only the gain in parsimony-given that intrinsic properties can be dispensed with in the ontology of physics - but also avoiding the costs that come with the commitment to quidditism, if the intrinsic properties are construed as categorical properties (as they have to be on Humeanism). George Darby enquires into the objections to quidditism and whether the shift to distance relations can really avoid quidditism. Whatever weight one may attribute to these objections, if a position can do without the commitment to quidditism, it is, other things being equal, preferable.

The commitment to quidditism arises if and only if there is a separation between the essence or nature of a property and the functional or causal role that it plays. However, there is no such separation in the case of distance relations. They are not causal relations. The dynamical structure, including the geometry, that a physical theory uses is introduced in terms of its causal or functional role for the change in the distance relations. But this implies that the distance relations themselves do not have a causal or functional role to play, on pain of an infinite regress of causal or functional roles. They are the bedrock, the primitive ontology, described by the basic, primitive notions; with respect to them all the other notions can then be introduced in terms of their functional role for them. Consequently, the issue of quidditism does not arise in the case of the distance relations. They are the only type of irreducible relations recognized in the Super-Humean ontology, and they do not admit of degrees. Furthermore, they are directly linked to the empirical evidence insofar as all the experimental evidence consists in the relative positions of discrete objects and the change of these positions. This, again, singles out the distance relations - although, as mentioned above, the argument for going for them as the ontological primitives is the overall coherence and explanatory fruitfulness of the resulting position. 


\section{Explanation}

Alastair Wilson - and to a certain extent also Anna Marmodoro - take the proposed ontology to be deficient for explanations. According to Wilson, this ontology leads to instrumentalism with respect to everything else apart from the ontological primitives. He is thus concerned with point (b) above: the proposed ontology may be sufficient, but it comes with a high cost for explanations that richer ontologies avoid. However, when defending the parsimonious ontology, the Super-Humean can adopt the same strategy as the standard Humean. There are patterns (stable regularities) in the change of the distance relations among the matter points, that is, their motion. Thus, some matter points move electronwise. Therefore, they are electrons and have negative charge. The only issue here is that being an electron and having negative charge are not intrinsic properties, but are derived from patterns in motion. But being derived from something else does not make charge unreal or a mere instrument for predictions. This is the same treatment that Humeans adopt, for instance, with respect to causation: it is not a relation between pairs of events; whether or not two events are related as cause and effect depends on a stable regularity.

As quoted in the introduction to the book, Jackson's recommendation for ontology is to "do with as meagre a diet as possible" $(1994,25)$. He then stresses that because ontology should be parsimonious, "there is bound to be a whole range of putative features of our world up for either elimination or location" $(1994,25)$. In our project, mass, charge, etc. are features of the world that are up for location, not elimination. If matter points, distance relations and change of distance relations are the basic notions, then everything else has to be located in configurations of matter points and their change. This is done via a functional definition of everything else in terms of its functional role for the change in the distance relations, which then enables us to locate gravitational mass, charge, etc. in certain patterns of motion. 
The same goes for the quantum state: it encodes also certain patterns of motion of quantum objects that become manifest in experimental contexts. It is true that the ontology of distance relations and their change only does not significantly restrict the kinematic possibilities for the matter points (since, for us, the geometry is part and parcel of the dynamical structure). The significant restrictions are the dynamical ones, that is, those coming from the dynamical structure. However, in contrast to what Wilson suggests, in order to be entitled to use these restrictions in explanations, one does not have to regard the state space as belonging to the fundamental ontology. It can be taken to be derived from the parsimonious ontology of matter points and change in their distance relations only by means of the location procedure that Jackson describes, namely by locating the restrictions that the state space imposes in the salient patterns of the evolution of the distance relations among the matter points.

George Darby is right in emphasizing that applying this view to the quantum state presupposes an ontology of quantum physics in terms of what is known as a primitive ontology of physical objects in ordinary space-time. The quantum state then is defined in terms of its functional role for the evolution of these objects. The argument for this stance and notably for spelling out the primitive ontology in terms of persistent particles - is that it offers the best solution to the measurement problem, as argued in the book. This argument is independent of the proposed ontology. It then shows how this ontology can be implemented in a precise physical theory of quantum mechanics, here in the guise of Bohmian mechanics. Once this argument is granted, there is indeed nothing fundamentally new or puzzling about the quantum state. It belongs to the dynamical structure, as do mass and charge. Consider the point that Bell $(1987,35)$ seeks to make when he says that "The electron need not turn out to be a small spinning yellow sphere". As Bell emphasizes, spin is not an intrinsic property of 
quantum systems, but a certain pattern of motion that shows up in certain experimental contexts.

Once we have identified the basic, salient regularities of particle motion, we have reached the bedrock. Indeed, the whole story could in principle be told by using only the notions of matter points, distance relations and change in distance relations; all explanations - including the one why salt dissolves in water - could in principle be framed by employing only these notions. But this would be inconvenient, for it would not flag the salient patterns in the change in the distance relations by using notions that lable precisely these patterns. That is what notions such as mass, charge, spin, quantum state, etc. do. Explanations that rely on them are explanations that show how particular phenomena come under these salient patterns (and one may then spell out these explanations in terms of unification, or in terms of - Humean causation). Taking these salient patterns for granted, the explanations in terms of these notions bring out constraints on what can and what cannot happen.

However, there is no scientific explanation of why there are the salient patterns of particle motion that there are in fact. If one wants notions such as mass, charge, spin, quantum state, etc. to yield such an explanation, one has to lift the status of these notions to referring to ingredients of the basic ontology that there are over and above matter points, distance relations and the change in these relations. But this is futile. These notions are defined in terms of their functional role for the particle motion. Consequently, explanations of why there are the salient patterns in the particle motion that there are in fact in terms of these notions would be circular. Thus, one does not explain why particles attract each other in terms of gravitational mass, since gravitational mass is defined in terms of its functional role for particle attraction, etc. Hence, there is no gain in explanation here. 
When it comes to ontology, taking these notions to refer to additional elements of the basic ontology only leads to well-known trouble. If mass, charge, spin, etc. are categorical, intrinsic properties of the particles, one runs into the trouble associated with quidditism and humility. If they are dispositional properties or powers, the problem is how they manifest themselves, notably how a power intrinsic to one particle can manifest itself in the acceleration of other particles. When it comes to the quantum state as represented by the wave function, the problem is that this state cannot be conceived as an entity that exists in physical space in addition to the particles and their motion, etc. In brief, a constraint in terms of irreducibly modal elements in the basic ontology over and above the particles and their motion does not lead to a gain in explanation and entails only trouble in the ontology. Any constraint that comes with ordinary scientific explanations is accounted for by us in terms of the mentioned location in (or identification of) salient patterns of particle motion. This assessment does not infringe upon us remaining finally neutral with respect to the metaphysics of modality. We only claim that the notions that scientists employ in formulating dynamical structures of physical theories do not reveal irreducibly modal elements of the world, but are located in salient patterns of particle motion. This may be considered as modesty with respect to what physics achieves; but it is not instrumentalism, and it fully respects the explanations that physics provides.

\section{Physics: quantum field theory}

When it comes to quantum field theory (QFT) by contrast to quantum mechanics, we agree with Adam Caulton that "No rigorous, realistic, relativistic quantum field theory (...) is known to exist". In fact, the situation is similarly bad for classical field theory. There, the only rigorously constructed models are either free of interaction (e.g. the evolution of Maxwell fields for prescribed currents) or describe charge densities that explode if no extra boundary conditions are supplied (e.g. Maxwell-Vlasov dynamics). The root of the problem is 
that most attempts in specifying a relativistic interaction are based on a notion of interaction between what occurs at space-time points, simply because the point is one of the few Lorentz invariant geometric objects - the other one being the hyperbola, which does not seem to be experimentally adequate. In order to describe radiation friction, however, it seems necessary to implement also an interaction at zero distances (one famous exception being WheelerFeynman electrodynamics). These so-called self-interaction terms are usualy ill-defined and their problems are directly inherited by the corresponding quantum field theories. Thus, strictly speaking, no rigorous, realistic field theory of interactions is known to exist, quantum or classical. In both cases, quantum and classical, this leaves those interested in ontology with two options: wait until such a theory will exist, or work out an ontology for the theory that we have at hand, however provisional such an ontology may be. We are concerned with the latter project in chapter 4 of the book. Our only aim in that chapter is to reply to the challenge that an ontology in terms of permanent matter points that are individuated by distance relations and their change is a non-starter when it comes to QFT, given notably the evidence of what is described as particle creation and annihilation phenomena. In other words, we cannot remain silent on QFT on pain of falling victim to this challenge.

Our choice of ontology for quantum physics is constrained by the measurement problem. We take an ontology of permanent particles with a law of motion for these particles (i.e. the Bohmian framework) to provide the best solution to the measurement problem. This problem hits QFT in the same way as non-relativistic quantum mechanics. This is the motivation for trying out the same solution for QFT. Hence, there is a positive reason to adopt the Bohmian ontology of permanent particles, namely that it is the best solution to the measurement problem. 
Thus, we set out to show that a Bohmian ontology of permanent particles with a deterministic law of motion for these particles can do the same for QFT (as it stands) as for quantum mechanics. This explains the choice of a particle ontology for QFT. No field ontology has been worked out that can account for the particle evidence in the experimental results. An ontology of both particles and fields would run into the well-known interaction problems, that is, would lift these problems from mathematical problems in the dynamical structure of all known field theories to problems in the basic ontology. (What comes closest to a field ontology is the GRWm ontology of a matter density field in quantum mechanics; but also the formalism of this theory is construed in terms of a fixed number of permanent particles. Furthermore, this ontology commits one to realism about wave function collapse with, in this case, the unpalatable consequence of having to countenance an instantaneous delocation of matter across arbitrarily big distances in space upon wave function collapse, as explained in chapter 3.3 of the book).

Against this background, it is clear that we are concerned with conventional, or cutoff quantum field theory (CQFT). This theory, by contrast to the algebraic, or axiomatic quantum field theory, and when subject to a well-defined and finite renormalization scheme, leads to a whole range of well-confirmed experimental predictions, given suitable cut-offs. Hence, this is the appropriate theory upon which to base the enquiry into an ontology of QFT. In our case we rely on the Dirac sea model that describes the electron sector of the standard model under the influence of external fields and which is also widely used to describe the next-generation experiments such as those ones planned at the ELI (Extreme Light Infrastructure). We then show how an ontology of a very large, but finite number of matter points that persist and that are subject to a deterministic law of motion results in the statistical predictions of CQFT, given appropriate cut-offs. In brief, as it is a mistake in quantum mechanics to infer from the Heisenberg uncertainty relations that there are no particle trajections, so it is a mistake to infer 
from the appearance and disappearance of particles in characteristic QFT experiments that there are no persistent particles. The commitment to persistent particles moving on trajectories that result from a deterministic law of motion provides in both cases a cogent ontology that accounts for the statistical predictions and experimental results. These are particle detection statistics.

Contrary to what Adam Caulton claims, $J$, the current, is an operator in QED and not a charge density valued field. It has to be sandwiched by states to form an expectation value, which then is about particle detection statistics. These particle detection statistics are what is measured, for instance, at CERN - precise energy deposits in precise solid angles with respect to the impact zone of the scattering experiment. The operator $J$ encodes the statistics of them. The ontology tells us about what these statistics are, namely about particle positions. In other words, the particles serve as truthmaker and explanation why we see a point and not a smeared out dot at the experiments in CERN. In the same vein also the electron and positron particle number operators are only operators and not numbers of particles. As we point out in the book, these do the bookkeeping of the statistics of the number of Fock excitations with respect to an arbitrarily chosen reference state. As the rigorous construction of the timeevolution of the Dirac sea model without cut-offs can only be implemented between timevarying Focks spaces - that is, with respect to time-varying reference states -, these operators constantly change their meaning over time (informally, they can be seen as undergoing timedependent Bogoliubov transformations). In fact, the electron and positron number opertors with respect to the fixed free vacuum state are in general even ill-defined on space-like Cauchy surfaces that intersect the support of the external influences. Hence, extreme caution is advisable when interpreting the statistics that these operators encode. Nevertheless, the proposed ontology provides the means to start developing detector models, that is, singling out appropriate detector reference states with respect to which the click statistics can be 
encoded by means of the corresponding particle number operators. The latter will prove to be a highly non-trivial and very technical task, which we did not dare to touch in this book.

Indeed, as Caulton says, the standard model of particle physics may turn out to be wrong well before we reach the Planck length. But then, when we have new physics, we have to go over the metaphysical books again. All that we claim in the book is that the commitments to matter points individuated by distance relations and the change in these relations provides a minimally sufficient ontology for the existing physics that accommodates all the explanatory power that this physics has.

\section{References}

Bell, John S. (1987): Speakable and unspeakable in quantum mechanics. Cambridge: Cambridge University Press.

Esfeld, Michael (1998): “Holism and analytic philosophy”, Mind 107, pp. 365-380.

Esfeld, Michael and Lam, Vincent (2011): “Ontic structural realism as a metaphysics of objects”. In A. and P. Bokulich (eds.): Scientific structuralism. Dordrecht: Springer. Pp. 143-159.

Jackson, Frank (1994): “Armchair metaphysics”. In: J. O’Leary-Hawthorne and M. Michael (eds.): Philosophy in mind. Dordrecht: Kluwer. Pp. 23-42.

Pettit, Philip (1993): The common mind. Oxford: Oxford University Press. 